\title{
Rossby vortex simulation on a paraboloidal coordinate system using the lattice Boltzmann method
}

\author{
Huidan $\mathrm{Yu}^{1,2,3, *}$ and Kaihua $\mathrm{Zhao}^{3}$ \\ ${ }^{1}$ State Key Laboratory of Nonlinear Mechanics, Institute of Mechanics, Chinese Academy of Sciences, Beijing 10080, China \\ ${ }^{2}$ Department of Physics, Zhejiang Normal University, Jinhua 321004, Zhejiang, China \\ ${ }^{3}$ Department of Physics and Center for Nonlinear Sciences, Peking University, Beijing 100871, China \\ (Received 2 October 2000; revised manuscript received 10 July 2001; published 16 October 2001)
}

\begin{abstract}
In this paper, we apply our compressible lattice Boltzmann model to a rotating parabolic coordinate system to simulate Rossby vortices emerging in a layer of shallow water flowing zonally in a rotating paraboloidal vessel. By introducing a scaling factor, nonuniform curvilinear mesh can be mapped to a flat uniform mesh and then normal lattice Boltzmann method works. Since the mass per unit area on the two-dimensional (2D) surface varies with the thickness of the water layer, the 2D flow seems to be "compressible" and our compressible model is applied. Simulation solutions meet with the experimental observations qualitatively. Based on this research, quantitative solutions and many natural phenomena simulations in planetary atmospheres, oceans, and magnetized plasma, such as the famous Jovian Giant Red Spot, the Galactic Spiral-vortex, the Gulf Stream, and the Kuroshio Current, etc., can be expected.
\end{abstract}

DOI: 10.1103/PhysRevE.64.056703

PACS number(s): 02.70.-c, 47.40.Nm, 51.10.+y

\section{INTRODUCTION}

In recent years, the lattice Boltzmann method (LBM) has developed into an effective numerical scheme for simulating fluid flows and modeling physics in fluid [1-4]. The scheme is particularly successful in fluid flow applications involving interfacial dynamics and complex boundaries. Meanwhile, the LBM has demonstrated a significant potential and broad applicability with numerous computational advantages, including the parallelism of algorithm, the simplicity of programming, and the ability to incorporate microscopic interactions. However, compared to the state-of-the-art of the conventional computational fluid dynamics technique, the LBM still suffers some limitations. One of them is the uniformity of its mesh grids. Historically, the LBM was developed from the lattice-gas automata method (LGAM) [5], consequently, the LBM inherits some features from its precursor. In the LGAM, the dynamics of particles evolving in a lattice space consists of two steps: Particles meeting at the same site collide according to a set of hard-sphere collision rules that conserve mass, momentum, and energy (for multispeed models) at each lattice site; after colliding, particles stream to the neighboring sites in the directions of their velocities. The small number of discrete velocities allowed are consistent with the lattice structure. In other words, the discretion of physical space is coupled with the discretion of momentum space. The lattice Boltzmann model was a direct transcription from the lattice-gas automata. The Boolean variables in the LGAM were replaced by real-number singleparticle distribution functions that eliminates the intrinsic stochastic noise in lattice-gas automata and subsequently enhance the computational efficiency. Two further improvements have been made to enhance the computational efficiency in the LBM already: The linearization of the collision operator [2] and the Bhatnagar-Gross-Krook (BGK) approximation [6] (which is also sometimes called single relaxation-

*Email address: h0y5840@aero.tamu.edu time approximation [3]). However, the uniformity of the lattice structure is persistent, which greatly hampers the broad application of the LBM because a curvilinear or irregular grid is much more efficient for many practical problems.

During the past few years, several researchers were motivated by such considerations to extend the applicability of the LBM to irregular lattices. Succi et al. [7] first proposed a finite-volume formulation of the lattice Boltzmann equation. Then, Chen [8] developed another elegant finite-volume scheme theoretically. Quite recently, H. Xi et al. [9] presented a simple but efficient finite-volume LBM from the point of view of modern finite-volume methods, which is applicable to unstructured meshes with arbitrary connectivity. However, the above-mentioned approaches of using irregular meshes are mostly theoretical research. Their applications need to be exploited. A scheme well applicable to long-term unsteady flow simulation, named as the interpolation-supplemented lattice Boltzmann equation (ISLBE) scheme was presented by X. He et al. [10]. In the ISLBE scheme, which is an LBM on polar coordinate system, a flow domain is discretized into an irregular mesh. At each node sits a regular velocity framework. Besides normal steps, relaxation, and streaming, the ISLBE includes an interpolation step to reconstruct the distribution function at grid nodes at the next step. Using the ISLBE scheme to simulate the two-dimensional (2D) vortex shedding phenomenon behind a circular cylinder for Reynolds numbers ranging from 50-150, the solutions are consistent with previous experimental observations and numerical simulations.

In this paper, we are going to apply our compressible LB model [11] to a rotating parabolic coordinate system. In order to simulate Rossby vortices emerging in a layer of shallow water flowing zonally in a rotating paraboloidal vessel, we need to treat a nonuniform curvilinear mesh. The crucial idea is to map the nonuniform curvilinear mesh to flat uniform mesh by rescaling all related physical quantities according to their dimensions using a scaling factor that is introduced to express a different linear scale at a different height along the curvilinear surface, and the different components of the distribution functions streaming between dif- 


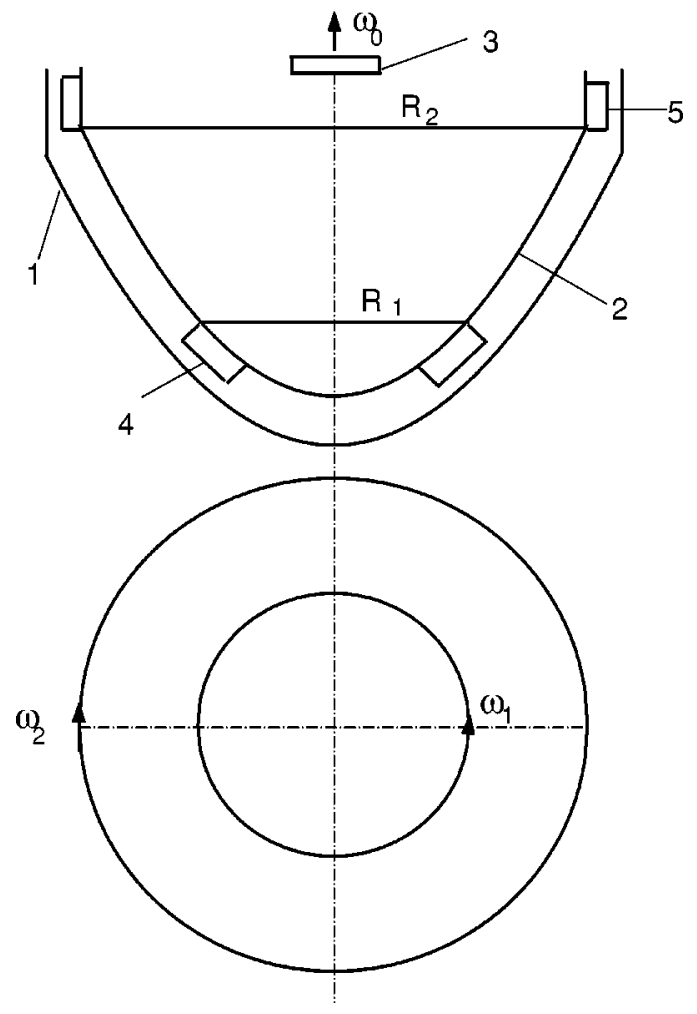

FIG. 1. Experimental configuration for producing Rossby vortices by zonal flow: (1) vessel with parabolic bottom; (2) liquid surface limited; (3) camera; $(4,5)$ rotating rings to create zonal flows. The vessel and the camera rotate at an angular rate.

ferent levels are modified accordingly. As a result, the scheme still consists of two steps: relaxation and streaming. Since the mass-per-unit area projected on the 2D surface varies with the thickness of the water layer, the $2 \mathrm{D}$ flow seems to be "compressible." We should regard the sound speed $c_{s}$ as the speed of surface wave in the present case. A compressible LBM scheme developed by us previously [11], is applied. Our simulation of Rossby vortices are consistent with the experimental observations reported by the Antipov and Nezlin and Fridman et al. groups $[12,13]$ qualitatively. The groups obtained a lot of experimental results about Rossby vortices, spiral structures, and solitons [14] that are closely related with many natural phenomena in planetary atmospheres, oceans, and magnetized plasma, such as the famous Jovian Giant Red Spot, the Galactic Spiral-vortex, the Gulf Stream, and the Kuroshio Current, etc.

\section{EXPERIMENTAL SETUP AND PARABOLOIDAL COORDINATES SYSTEM}

Figure 1 shows the sketch of the experimental setup. A revolutionary paraboloidal vessel is rotating with angular velocity $\omega_{0}$, a layer of water of uniform thickness is formed in the vessel when gravity is balanced by the centrifugal force. As the thickness of the water layer is small compared with the linear dimension of its surface, the simulation can be treated as a $2 \mathrm{D}$ problem. Shearing flow is driven by a pair of rings with differential rotation speed $\omega_{1}$ and $\omega_{2}$ at the radii $R_{1}$ and $R_{2}$, respectively. It becomes unstable and vortices are produced when certain critical strength of the differential rotation is attained. The perturbed layer is no longer of uniform thickness. Density $\rho$ is defined as the mass of water-per-unit area projected normally to the curved surface of the vessel, it varies from site to site as well as with time $t$.

The band region between radii $R_{1}$ and $R_{2}$ is covered with a nonuniform paraboloidal mesh (see Fig. 2) which divides the circumstance into $N$ grids throughout the bandwidth from the bottom to the top. The curvilinear mesh so obtained is nonuniform and the edge length $a$ of the grids increases with the level height.

In order to match the experimental solutions, we must deal with our simulations on a rotating parabolic coordinate system (Fig. 3). The relations between the Cartesian coordinates $(x, y, z)$ and the paraboloidal coordinates $(\eta, \zeta, \phi)$ are

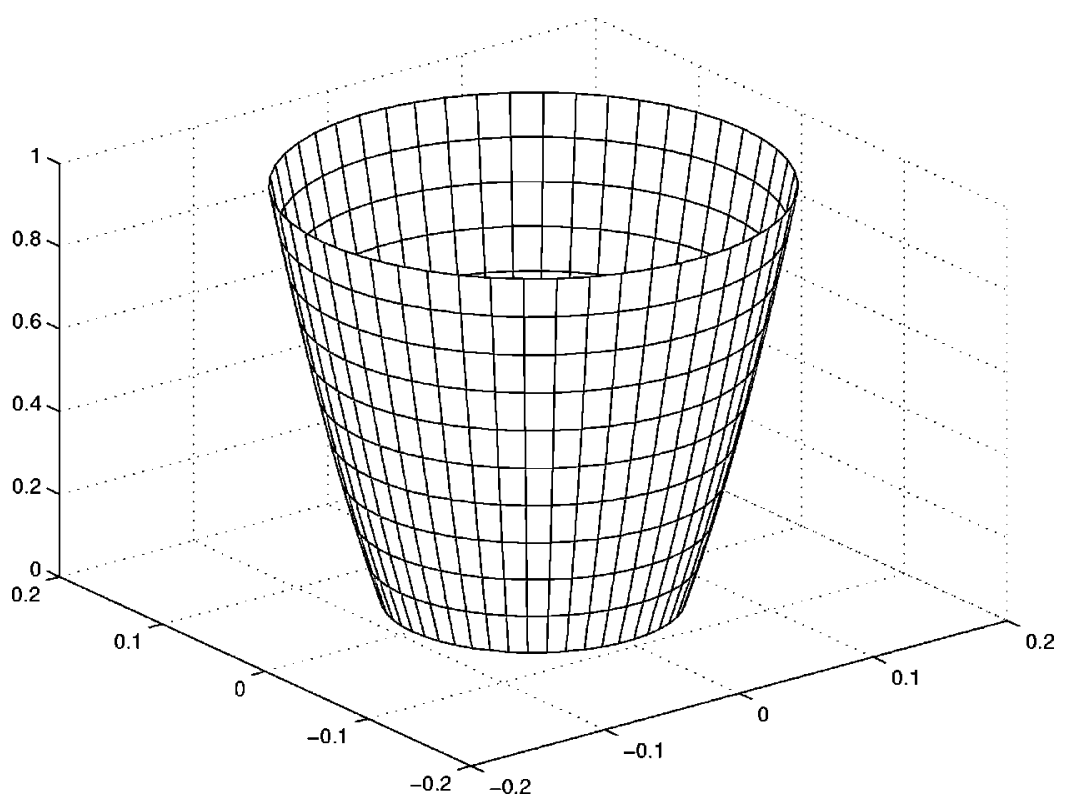

056703-2
FIG. 2. Nonuniform curvilinear mesh. 


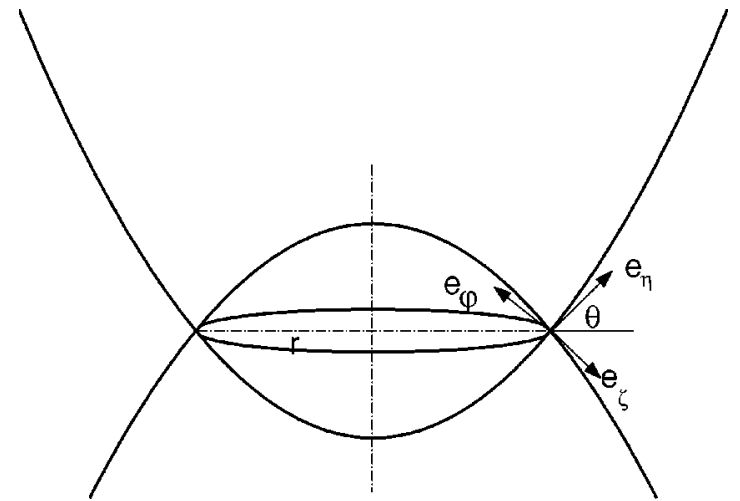

FIG. 3. Paraboloidal coordinate system and its unit vectors.

$$
\begin{gathered}
x=\eta \zeta \cos \phi, \\
y=\eta \zeta \sin \phi, \\
z=\frac{1}{2}\left(\eta^{2}-\zeta^{2}\right) .
\end{gathered}
$$

Let $\vec{u}$ be the velocity of the water in the frame of reference corotating with the vessel. When $\vec{u}=0$, the tangential component of gravity is balanced by tangential part of the centrifugal force. When $\vec{u} \neq 0$, the resultant force $\vec{F}$ exerting on the water consists of the Coriolis force and the part of the centrifugal force with quadratic dependence on the components of $\vec{u}$, i.e.,

$$
\begin{aligned}
& F_{\phi}=2(\sin \theta) u_{\eta} \omega_{0}-\frac{(\sin \theta) u_{\phi} u_{\eta}}{r}, \\
& F_{\eta}=-2(\sin \theta) u_{\phi} \omega_{0}+\frac{(\sin \theta) u_{\phi}^{2}}{r},
\end{aligned}
$$

with

$$
\sin \theta=\frac{p}{\sqrt{r^{2}+p^{2}}},
$$

where $\mathrm{p}$ is the focus length of the paraboloid, $\omega_{0}=\sqrt{g} / \zeta_{0}$ the angular speed of the rotating parabolic system, $\zeta=\zeta_{0}=p^{2}$ is the equation of the paraboloidal vessel surface.

\section{LATTICE BOLTZMANN METHOD FOR “COMPRESSIBLE”' 2D FLOWS}

Since the mass-per-unit area projected on the 2D surface varies with the thickness of the water layer, the $2 \mathrm{D}$ flow seems to be "compressible." We should regard the sound speed $c_{s}$ as the speed of surface wave in the present case.

The LBM for compressible flow developed by us in [11] is applied at first to a uniform hexagonal grid on a rectangular domain with $\mathrm{N}$ cells horizontally connected by cyclic boundary condition. We take the FHP seven-bit model: $\left|\vec{e}_{a}\right|$ $=1$ for $a=1, \ldots, 6$, and $\left|\vec{e}_{0}\right|=0$. The equation of the evolu- tion of the distribution function is

$$
f_{a}\left(\vec{x}+\vec{e}_{a}, t+1\right)=f_{a}^{*}(\vec{x}, t)+\Omega_{a}^{*}(\vec{x}, t),
$$

where the collision term assumes the form

$$
\Omega_{a}^{*}=-\frac{1}{\tau}\left(f_{a}^{*}-f_{a}^{* e q}\right),
$$

and

$$
\begin{gathered}
f_{a}^{*}(\vec{x}, t)=f_{a}(\vec{x}, t)+\Phi_{a}(\vec{x}, t)+F_{a}(\vec{x}, t), \\
\Phi_{a}=\frac{1}{3} \vec{e}_{a} \cdot g c_{s}^{2} \vec{\nabla} \rho, \\
F_{a}=\frac{1}{3} \vec{e}_{a} \cdot \vec{F} .
\end{gathered}
$$

$\Phi_{a}$ is an attractive force introduced in order to reduce the wave speed $c_{s}$ to $c_{s}^{*}=\sqrt{1-g} c_{s}$ in which $g$ is an adjustable parameter. $\vec{F}$ is the resultant force exerting on the simulated system, the components of which are given in Eqs. (4) and (5) above. The equilibrium distribution functions $f_{a}^{* e q}$ in Eq. (7) are taken as

$$
\begin{gathered}
f_{a}^{* e q}=\rho\left[c_{s}^{2}+\vec{e}_{a} \cdot \vec{u}^{*}+2\left(\vec{e}_{a} \cdot \vec{u}^{*}\right)^{2}-\vec{u}^{* 2}\right], \\
f_{0}^{* e q}=\rho\left(1-2 c_{s}^{2}-\vec{u}^{* 2}\right),
\end{gathered}
$$

here, $\rho^{*}$ and $\vec{u}^{*}$ are defined as

$$
\begin{gathered}
\rho^{*}=\sum_{a=0}^{6} f_{a}^{*}=\rho, \\
\rho^{*} \vec{u}^{*}=\sum_{a=1}^{6} \vec{e}_{a} f_{a}^{*}=\rho \vec{u}+g c_{s}^{2} \vec{\nabla} \rho+\vec{F},
\end{gathered}
$$

with

$$
\begin{gathered}
\rho=\sum_{a=0}^{6} f_{a}, \\
\rho \vec{u}=\sum_{a=1}^{6} \vec{e}_{a} f_{a},
\end{gathered}
$$

and

$$
\begin{gathered}
\sum_{a=0}^{6} \Phi_{a}=\sum_{a=0}^{6} F_{a}=0, \\
\sum_{a=0}^{6} \vec{e}_{a} \Phi_{a}=g c_{s}^{2} \vec{\nabla} \rho, \quad \sum_{a=0}^{6} \vec{e}_{a} F_{a}=\vec{F} .
\end{gathered}
$$

By the Chapmann-Enskog expansion for the $\vec{e}_{a}$ 's zeroth and first moments of Eq. (6), we obtain 


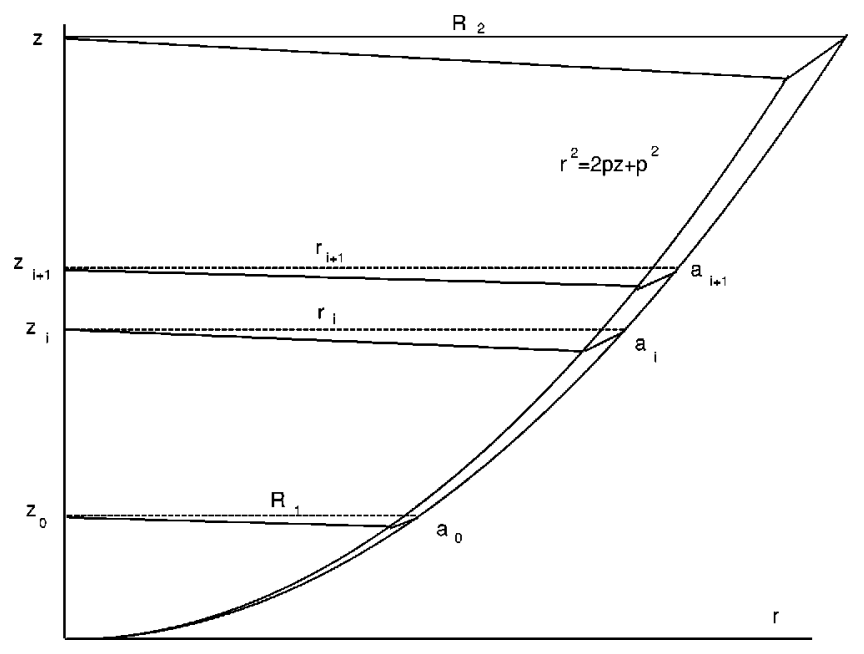

FIG. 4. Recursive relation of scaling factor.

$$
\begin{gathered}
\frac{\partial \rho}{\partial t}+\vec{\nabla} \cdot(\rho \vec{u})=0 \\
\rho\left[\partial_{t} \vec{u}+(\vec{u} \cdot \vec{\nabla}) \vec{u}\right]=-c_{s}^{* 2} \vec{\nabla} \rho+\vec{\nabla}^{2}(\eta \vec{u})+\vec{\nabla}[\vec{\nabla} \cdot(\zeta \vec{u})]+\vec{F},
\end{gathered}
$$

with $c_{s}^{*}=\sqrt{1-g} c_{s}$ effective sound speed for compressible flows.

\section{MAPPING THE NONUNIFORM CURVILINEAR MESH ONTO A UNIFORM FLAT MESH BY RESCALING}

In order to use the normal LBM to simulate the Rossby vortices in a zonal band on a paraboloidal surface with a nonuniform mesh, we cut the band along the $\eta$ direction and map it onto a rectangle with uniform mesh. Since the edge length of grids in the uniform mesh corresponds to different scales at different heights in the curvilinear mesh, all the physical quantities, such as density, velocity, etc., should be rescaled accordingly.

Let $a_{j}$ be the edge length of the nonuniform curvilinear meshes in the $j$ th horizontal line counted from bottom (Fig. 4). We can derive a recursion formula for the scaling ratio from the configuration of treated mesh.

$$
\frac{a_{j+1}}{a_{j}}=1+\frac{2 \pi \beta}{N \sqrt{1+\left(r_{j} / p\right)^{2}}},
$$

with $\beta$ the height-to-edge ratio, which is $\sqrt{3} / 2$ for hexagonal grid. Taking the lower boundary $r_{0}=R_{1}, a_{0}=2 p R_{1} / N$ as datum, we can calculate $a_{1}, a_{2}, \ldots$, one by one by Eq. (17) up to the upper boundary $a_{N_{Y}}$.

All macrophysical quantities such as density, momentum, and wave speed in our simulation should be multiplied by a scaling factor according to their length dimension, e.g., $\rho_{j}$ $\rightarrow \rho_{j} a_{j}^{2}, c_{s j} \rightarrow c_{s j} / a_{j}$, etc., where $j=0,1,2,3, \ldots$, refers to the numbering of the horizontal levels. Besides, distribution functions should be rescaled at certain stages. Namely, when streaming, distribution functions from level $j+1$ to level $j$ are multiplied by factor $a_{j}^{2} / a_{j+1}^{2}$ and $a_{j}^{2} / a_{j-1}^{2}$ from the $j$
-1 level to the $j$ level. The same treatment must be done for $\vec{\nabla} \rho$ in Eq. (13) which is discretized as (4/9) $g c_{s}^{2}\left\{\rho\left(\vec{x}+\vec{e}_{a}\right)\right.$ $\left.-\rho\left(\vec{x}-\vec{e}_{a}\right)-(1 / 8)\left[\rho\left(\vec{x}+2 \vec{e}_{a}\right)-\rho\left(\vec{x}-2 \vec{e}_{a}\right)\right]\right\}^{(11)}$.

The effect wave speed $c_{s}^{*}=c_{s} \sqrt{1-g}$ should be scaled as

$$
\frac{c_{s j}^{*}}{c_{s 0}^{*}}=\frac{a_{0}}{a_{j}}
$$

We accomplish this by scaling of $\mathrm{g}$ instead of $c_{s}$

$$
g_{j}=1-\frac{1-g_{0}}{a_{j}^{2}},
$$

where $g_{0}$ is $g$ at level $j=0$.

Appropriate $g_{0} \lesssim 1$ is chosen to get the effective $c_{s}^{*}$ needed in simulations. In this paper, we choose $g_{0}=0.99$.

Now the iteration procedures described in Sec. II are carried on as if we are working on a flat uniform mesh.

\section{RESULTS OF SIMULATION}

All our simulations are conducted according to the experimental setup shown in Fig. 1. The vessel's dimensions are: $R_{1}=0.05 \mathrm{~m}, R_{2}=0.15 \mathrm{~m}, p=0.0817 \mathrm{~m}$. The vessel and camera rotate at a fixed angular rate $\omega_{0}=10.99 \mathrm{rad} / \mathrm{s}$ (clockwise). Both the angular speed and sense of rotation of zonal flows $\left(\omega_{1}, \omega_{2}\right)$ are adjustable.

Our simulations are carried on in a corotating frame with the vessel.

Set $N_{X}=498$ sites along circumstance of the vessel. From the recursion formula (17), $N_{Y}=153$ is generated along the surface of the vessel. The speed of surface wave is assumed $0.5 \mathrm{~m} / \mathrm{s}$ and $c_{s}=0.52$ so that the relation between lattice unit and real physical unit is established

$$
l_{0}=\frac{2 \pi R_{2}}{N_{X}}=0.001907 \mathrm{~m} / l \text {, }
$$

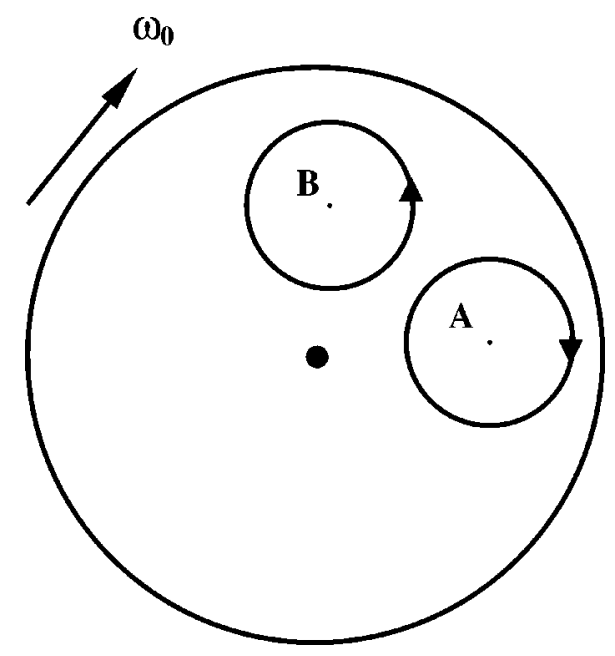

FIG. 5. A schematic illustration of cyclone $(A)$ and anticyclone (B). 


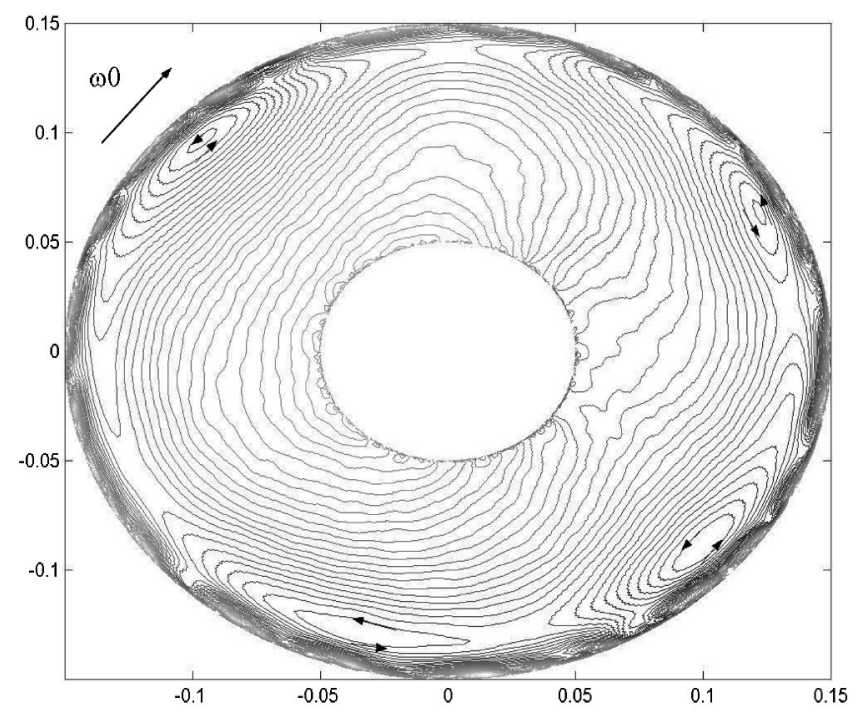

FIG. 6. Four anticyclonic Rossby vortices generated by the anticyclonic shear flow that $\omega_{2}=10.99 \mathrm{rad} / \mathrm{s}$ (counterclockwise) and $\omega_{1}=4.82 \mathrm{rad} / \mathrm{s}$ (clockwise), 50000 time steps, then $\omega_{1}$ is increased to $17.28 \mathrm{rad} / \mathrm{s}$ (clockwise), 15000 time steps more, totally 65000 time steps.

$$
t_{0}=\frac{2 \pi c_{s} R_{2} \sqrt{\left(1-g_{0}\right)}}{N_{X} v_{s}}=0.000198 \mathrm{~s} / t
$$

where $\mathrm{m} / l$ and $\mathrm{s} / t$ mean meters per lattice and seconds per time step, respectively.

All simulations solutions shown below are projection drawings from upper to down and correspond to stationary. The time steps in them can be transfered to be real physical units via Eq. (21).

At the lower boundary $j=0$ and the upper boundary $j$ $=N_{Y}$, we force the density fixed to $\rho=\rho_{0}=1$ and the velocities fixed to $u_{1}=2 \pi R_{1}\left(\omega_{1}-\omega_{0}\right)$ and $u_{2}=2 \pi R_{2}\left(\omega_{2}\right.$

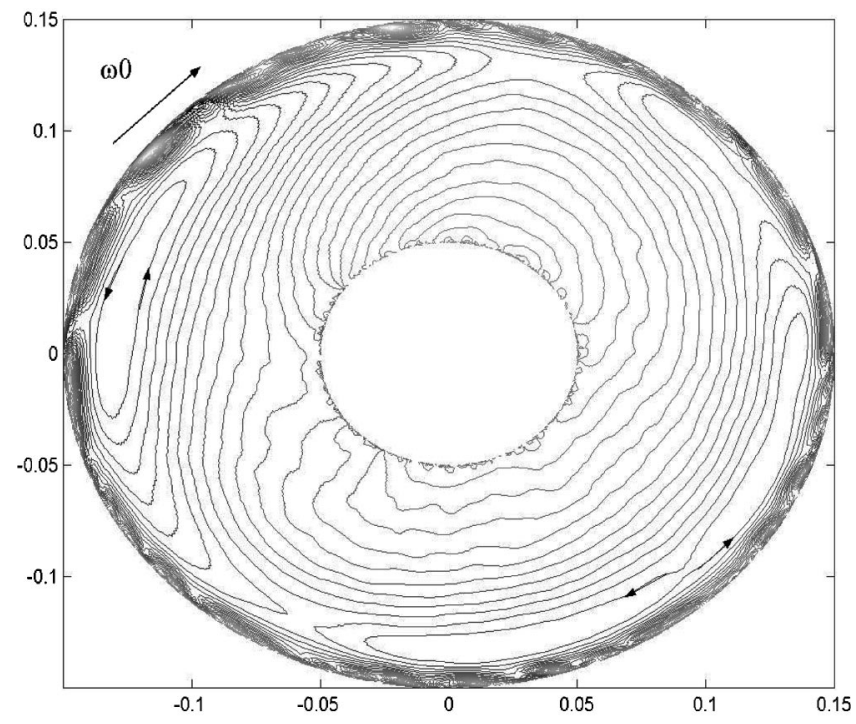

FIG. 7. Two anticyclonic Rossby vortices generated by the anticyclonic shear flow $\omega_{2}=10.99 \mathrm{rad} / \mathrm{s}$ (counterclockwise) and $\omega_{1}$ $=17.28 \mathrm{rad} / \mathrm{s}$ (clockwise), 650000 time steps.

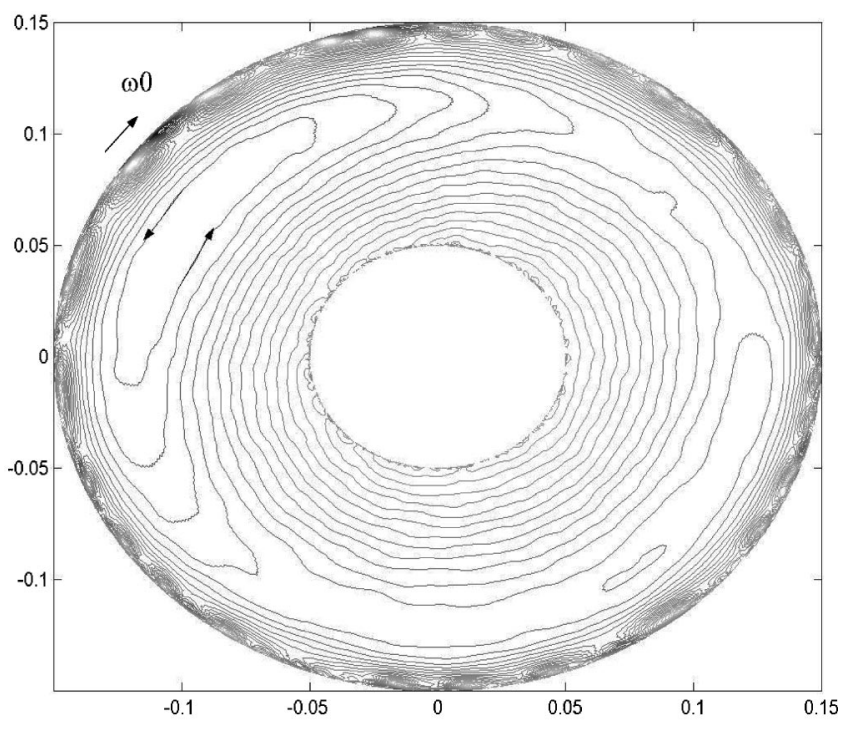

FIG. 8. Single anticyclonic Rossby vortex generated by the anticyclonic shear flow $\omega_{2}=5.24 \mathrm{rad} / \mathrm{s}$ (counterclockwise) and $\omega_{1}$ $=17.28 \mathrm{rad} / \mathrm{s}$ (clockwise).

$\left.-\omega_{0}\right)$, respectively. The periodic boundary condition is taken at the left-side and right-side boundaries. $\rho=\rho_{0}=1$ and $u$ $=u_{0}=0$ are put at all the inner sites initially.

The Rossby vortices emerge in the flow driven by the shearing velocities of the upper and lower boundaries.

By definition [14] (see schematic illustration in Fig. 5), if the vorticity of zonal flows is parallel to the local component of the angular velocity, the vorticity $(A)$ is cyclonic; otherwise, the vorticity $(B)$ is anticyclonic.

The following phenomena were reported by the Nezlin and Snezhkin group in their experiments [14].

(1) If the zonal flow is anticycolonic, the vortices produced are accordingly anticyclones;

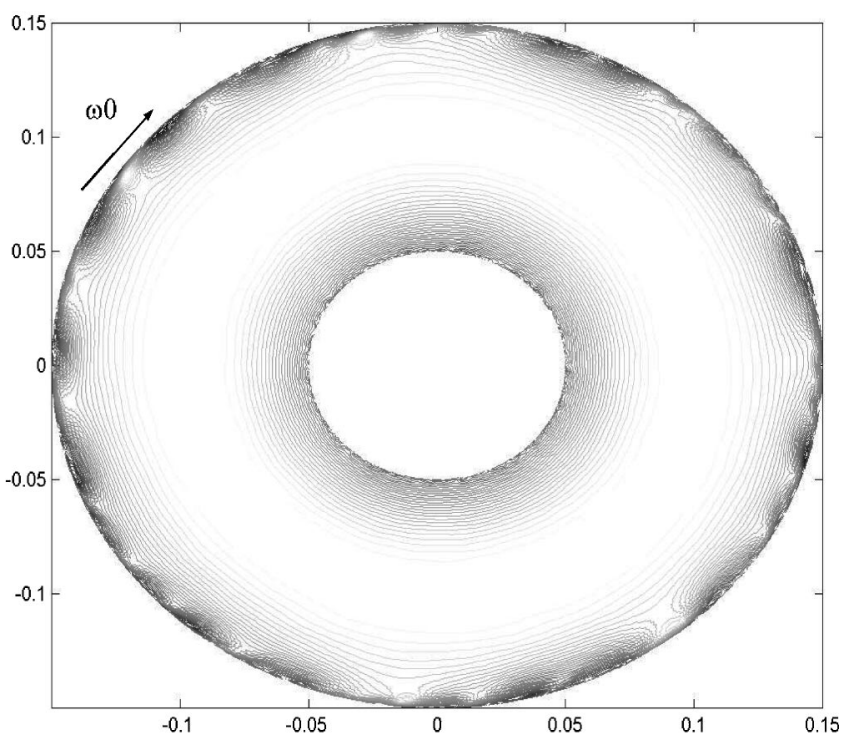

FIG. 9. No vortex generated by the cyclonic shear flow $\omega_{2}$ $=7.33 \mathrm{rad} / \mathrm{s}$ (clockwise) and $\omega_{1}=21.99 \mathrm{rad} / \mathrm{s}$ (counterclockwise). 
(2) If the zonal flow is cyclonic, no large-scale vortex is generated.

In our simulations we found the following phenomena: Four anticyclonic Rossby vortices are produced by two anticyclonic shear flows after 65000 time steps, in which 50000 steps were run with $\omega_{2}=10.99 \mathrm{rad} / \mathrm{s}$ (counterclockwise) and $\omega_{1}=4.82 \mathrm{rad} / \mathrm{s}$ (clockwise), followed by 15000 steps with $\omega_{1}$ increased to $17.28 \mathrm{rad} / \mathrm{s}$ (clockwise) (Fig. 6); however, two anticyclonic Rossby vortices are produced after 65000 time steps if $\omega_{1}=17.28 \mathrm{rad} / \mathrm{s}$ (clockwise) from the begining (Fig. 7). That is to say, the final state of vortical flow is history dependent.

In another case, single anticyclonic Rossby vortex are produced by a shear flow $\omega_{2}=5.24 \mathrm{rad} / \mathrm{s}$ (counterclockwise) and $\omega_{1}=17.28 \mathrm{rad} / \mathrm{s}$ (clockwise) (Fig. 8).

No vortex produced by a cyclonic shear flow $\omega_{2}$ $=7.33 \mathrm{rad} / \mathrm{s}$ (clockwise) and $\omega_{1}=21.99 \mathrm{rad} / \mathrm{s}$ (counterclockwise) (Fig. 9).

The results of our simulation are as follows.

(1) When the shear flow is anticycolonic, the vortices produced are, accordingly, anticyclones (Figs. 6, 7, 8), and the vortex number is history dependent, as shown in Figs. 6 and 7.
(2) When the shear flow is cyclonic, no vortex emerges (Fig. 9), which agrees with the experimental solutions from the Antipov and Nezlin group.

\section{CONCLUSION}

By introducing scaling factors, we generalize the LBM from uniform flat mesh to nonuniform curvilinear mesh. Using the LBM for compressible flows developed by us, we have simulated the Rossby vortices on a rotating parabolic Coordinate frame and the results are consistent with existing experimental observations qualitatively, which encourages us to explore more quantitative solutions relating to Rossby vortices and simulate more complicated experimental phenomena, such as drift vortices and solitons both in nature and in magnetized plasma, galactic spiral structures, etc.

\section{ACKNOWLEDGMENTS}

We especially thank Dr. Hudong Chen for his instructive discussions and also wish to thank Professor Mengfen Xia and Professor Yaosong Chen for their valuable ideas and suggestions.
[1] G. McNamara and G. Zanetti, Phys. Rev. Lett. 61, 2332 (1988).

[2] F.J. Higuera, S. Succi, and R. Benzi, Europhys. Lett. 9, 345 (1989); F.J. Higuera and J. Jemenez, ibid. 9, 663 (1989).

[3] H. Chen, S. Chen, and W.H. Matthaeus, Phys. Rev. A 45, R5339 (1991); Y. Qian, D. d'Humieres, and P. Lallemand, Europhys. Lett. 17, 479 (1992).

[4] S. Chen and G. Doolen, Annu. Rev. Fluid Mech. 30, 329 (1998).

[5] U. Frisch, B. Hasslacher, and Y. Pomeau, Phys. Rev. Lett. 56, 1505 (1986); S. Wolfram, J. Stat. Phys. 45, 471 (1986).

[6] P.L. Bhatnagar, E.P. Gross, and M. Krook, Phys. Rev. 94, 511 (1954).
[7] S. Succi, G. Amati, and R. Benzi, J. Stat. Phys. 81, 5 (1995); F. Nannelli and S. Succi, ibid. 68, 401 (1998).

[8] H. Chen, Phys. Rev. E 58, 3955 (1998).

[9] H. Xi, G. Peng, and S. Chou, Phys. Rev. E 59, 6202 (1999).

[10] X. He and G. Doolen, Phys. Rev. E 56, 434 (1997).

[11] H. Yu and K. Zhao, Phys. Rev. E 61(4), 3867 (2000).

[12] S.V. Antipov, M.V. Nezlin, E.N. Snezhkin, and A.S. Trubnikov, Nature (London) 323, 238 (1986).

[13] A.M. Fridman, A.G. Morozov, M.V. Nezlin, and E.N. Snezhkin, Phys. Lett. 109A, 228 (1985).

[14] M. V. Nezlin and E. N. Snezhkin, Rossby Vortices, Spiral Structures, Solitons (Astrophysics and Plasma Physics in Shallow Water Experiments) (Springer-Verlag, Heidelberg, 1993). 